\title{
Developing improved tissue-engineered buccal mucosa grafts for urethral reconstruction
}

\author{
A. Simsek ${ }^{1,2}$; A.J. Bullock ${ }^{2}$; S. Roman ${ }^{2}$; C.R. Chapple ${ }^{1}$; S. MacNeil ${ }^{2}$ \\ ${ }^{1}$ Royal Hallamshire Hospital, Department of Female and Reconstructive Urology, \\ Sheffield; ${ }^{2}$ University of Sheffield, Department of Materials Science \& Engineering, Sheffield; United \\ Kingdom
}

Funding/Support: A. Simsek was funded by the Scientific and Technological Research Council of Turkey.

Cite as: Can Urol Assoc J 2018 Feb. 6; Epub ahead of print. http://dx.doi.org/10.5489/cuaj.4826

Published online February 6, 2018

\section{Abstract}

Introduction: We aimed to compare alternative synthetic scaffolds suitable for future implantation and to examine the use of an inhibitor of lysyl oxidase (beta-aminopropionitrile $[\beta-\mathrm{APN}])$ to reduce contraction in these implants.

Methods: Three synthetic scaffolds were compared to natural dermis as substrates for the production of tissue-engineered skin. For natural dermis, Euroskin was used to provide a cell-free cadaveric dermis. Synthetic scaffolds consisted of microfibrous PLA, nanofibrous PHBV, and a micro-/nanofibrous trilayer of PLA-PHBV-PLA. The latter were all electrospun and then all four scaffolds (three synthetic, one natural) were placed in six well plates. A culture well was formed on the scaffold using a $1 \mathrm{~cm}$ diameter stainless steel ring and $1.5 \times 10^{5}$ oral fibroblasts were seeded one side; after two days of culture, the ring was placed on the other side of the scaffolds and $3 \times 10^{5}$ oral keratinocytes were seeded on to the scaffolds and cultured with keratinocytes uppermost. After a further two days of culture, scaffolds were cut to $1 \mathrm{~cm}^{2}$ and raised to an air-liquid interface on stainless steel grids and some were treated with 200 $\mu \mathrm{g} / \mathrm{mL}$ beta-aminopropionitrile (BAPN) throughout the culture period (28 days). Contraction in vitro was assessed by serial digital photography of cell-seeded scaffolds and cell-free scaffolds three times a week for 28 days. All cell-seeded scaffolds were assessed for cell metabolic activity, mechanical properties, histology, and morphology by scanning electron microscopy (SEM).

Results: The mean fibre diameters and pore sizes of PLA and PHBV scaffolds were $2.4 \pm 0.77,0.85 \pm 0.21 \mu \mathrm{m}(\mathrm{p}<0.001)$, and $10.8 \pm 2.3,4.3 \pm 1.1 \mu \mathrm{m}(\mathrm{p}<0.001)$, respectively. Oral fibroblasts and keratinocytes were tightly adhered and grew well on both surfaces of trilayer. The ultimate tensile strength (UTS) and Young's modulus (YM) 
of PLA samples were significantly lower than Euroskin $(\mathrm{p}<0.001$ and $\mathrm{p}<0.05$, respectively); only the UTS of the trilayer samples was slightly significantly lower $(p<0.05)$. Metabolic activity was significantly increased for cells on all scaffolds without significant differences between them from Day 0 to Day 28. There were no adverse effects of $\beta$-APN on cell viability. With respect to contraction, cells on trilayer and PHBV monolayers did not undergo any significant contraction; however, cells on PLA monolayer and Euroskin contracted $25.3 \%$ and 56.4\%, respectively, over 28 days. The addition of $200 \mu \mathrm{g} / \mathrm{ml} \beta$-APN significantly reduced contraction of Euroskin compared with the control $(\mathrm{p}<0.01)$; however, $\beta$-APN did not affect PLA contraction during this culture period ( $p>0.05$ ).

Conclusions: This study shows that a trilayer micro-nano-3D porous synthetic scaffold is suitable for oral keratinocyte and fibroblast growth with good cell viability and minimal contraction. This material also has good mechanical properties and histological analyses showed its ability to mimic normal human oral mucosal morphology. Furthermore, synthetic trilayer scaffolds have advantages over biological scaffolds - there is no risk of disease transmission or immunological rejection and they appear resistant to contraction. We suggest they present a good alternative to allodermis for future use in urethral reconstruction.

\section{Introduction}

Urethral strictures are an abnormal narrowing of the urethra which can be due to iatrogenic, idiopathic, inflammatory or traumatic causes. The most common etiology of urethral stricture is idiopathic (41\%) followed by iatrogenic (35\%). Iatrogenic injuries are related to placing of indwelling catheters, transurethral manipulation, surgery for hypospadias, prostatectomy, and brachytherapy $(1,2)$.

Overall the incidence of urethral strictures is about $1 \%$ for males over the age of 55 . The actual incidence differs based on worldwide populations, geography, and income $(3,4)$.

Treatment of urethral strictures depends on stricture aetiology, localisation, stricture length, the degree of spongiofibrosis, the previous history of treatment, and the patient's age. Short simple strictures are treated endoscopically, however longer more complex strictures in the penile urethra often require a one or two stage urethroplasty. In carrying out an augmentation procedure, a range of materials have been used for grafting including penile skin, scrotal skin, oral mucosa, bladder mucosa, and colonic mucosa. From these, oral mucosa grafts have become the most clinically effectivedue to their short harvest time, lack of hair, low associated morbidity, and high clinical success rates (5-8). Full-thickness oral mucosa is taken as most patients can provide an adequate donor area.

Reported complications of oral mucosal grafts include intra-operative haemorrhage, post-operative infection, pain, swelling, and damage to salivary ducts $(9,10)$. In particular it can be difficult to obtain enough buccal mucosa for long grafts. Accordingly to reduce donor site morbidity tissue engineered buccal grafts have been 
used as an alternative for reconstructive of complex urethral strictures.

In 2004 (11) our group developed TE buccal mucosa (TEBM) based on allogeneic cadaveric dermal tissue and autologous cultured buccal mucosa keratinocytes and fibroblasts for reconstruction of the urethra . In 2008 we reported the 3 year outcomes of a first clinical trial of this material in five patients $(11,12)$ We found that initial results were good in all five patients with rapid vascularisation of the grafts allowing successful retubularisation of all patients as though native buccal mucosa had been used. However after 8 and 9 months respectively two of the five patients' grafts developed contraction and fibrosis-this affected part of the graft for one patient and all of the graft for another Our 9 year follow up of these patients published in 2014 showed no further fibrosis for the 4 patients who still had TE buccal mucosa in place (13).

We have more recently looked beyond using native dermis as a scaffold for tissue engineered of buccal mucosa for two reasons-eliminating any risk of infection and reducing graft contracture. With donor grafts there is always a potential, albeit small risk, of disease transmission. This can be mitigated by accessing cadaveric skin from accredited tissue banks where donors are screened for bacteria and for Hepatitis $\mathrm{B}$ and $\mathrm{C}$ and efforts are made to exclude patients carrying HIV - the latter is the most challenging as there is a period of seroconversion in which a patient may be infected and yet not test positive. Clearly with cadaveric donation this will always remain a small risk.

The other reason that we have moved to synthetic dermal substrates is to see if we can overcome some of the problems of graft contraction and fibrosis. Therefore we developed synthetic electrospun biodegradable scaffolds for urethral reconstruction (14). We have also explored using drug approaches to prevent contraction of dermal based scaffolds used in tissue engineered skin production (15). In this study we have explored the production of tissue engineered buccal mucosa using de-epidermised acellular dermis, in this case sourced from an accredited tissue bank (Euro Skin) and compared it to buccal mucosa based on three synthetic scaffolds, PLA, PHBV and a trilayer scaffold which consists of microfibers of PLA and nanofibers of PHBV.

Poly-L-lactic acid (PLA) has a long history of use and is well known to be a biodegradable biomaterial with good biocompatibility $(16,17)$. It has been used extensively in our group (18-21) for applications ranging from a material for repair of the pelvic floor (21) to a combination of PLA with polyglycolic acid (PGA), as PLGA, to make a biodegradable scaffold for use in the cornea (22). The second polymer we used namely, Poly(3-hydroxybutyrate-co-3-hydroxyvalerate) (PHVB) was selected because it can be readily spun into nanofibrous layers as we have shown (23) and when combined with microfibers of PLA can produce a microporous/nanoporous/microporous trilayer which mimics to some extent the architecture of skin in that the PHBV fibres can be produced as a thin compact layer acting to some extent as a pseudo- basement membrane. This allows the segregation of different cell types during which we have recently published $(23,24)$ 
Accordingly the aim of this study was to improve on the development of tissue engineered buccal mucosa for future clinical use by comparing a synthetic trilayer (PLA-PHBV-PLA) biodegradable alternative to human dermis and further to explore the use of beta-aminopropionitrile ( $\beta$-APN) as a lysyl oxidase inhibitor to reduce contraction in these grafts.

\section{Methods}

Preparation of PLA and PHBV monolayer scaffolds

Poly-L-lactic acid ((PLA) Goodfellow, Cambridge, UK) was dissolved in dichloromethane (DCM) at 10\% wt PLA, PHBV (Goodfellow, Huntingdon, UK) was dissolved in $90 \mathrm{wt} \% \mathrm{DCM} / 10 \mathrm{wt} \%$ methanol solution. For random scaffolds polymer solutions were loaded into $5 \mathrm{~mL}$ syringes fitted with blunt tipped stainless steel needles with an internal diameter of $0.8 \mathrm{~mm}$ (I\&J Fisnar Inc.). Four syringes with $5 \mathrm{~mL}$ solution in each were used to deliver the solutions at a constant flow rate of $2.4 \mathrm{~mL} / \mathrm{h}$ using a programmable syringe pump (Genic, Kent Scientific, USA). Solutions were electrospun horizontally with an accelerating voltage of $17 \mathrm{kV}$ supplied by a high voltage power supply (Model 73030 P, Genvolt, Shropshire, UK). Fibrous mats were collected on aluminium foil sheets $(18 \mathrm{~cm} \times 16 \mathrm{~cm})$ wrapped around an earthed aluminium rotating collector (rotating at $300 \mathrm{r} / \mathrm{min}$ ) $17 \mathrm{~cm}$ (PLA), $10 \mathrm{~cm}$ (PHBV) from the tip of the needle.

\section{Preparation of PLA-PHBV-PLA trilayer scaffold}

Micro-fibrous PLA and nano-fibrous PHBV trilayer scaffolds were electrospun using parameters as described by Bye et al (23). PLA and PHBV solution were pumped from 4 syringes at $2.4 \mathrm{~mL} / \mathrm{h}$ per syringe. For PLA a needle to collector distance of 17 $\mathrm{cm}$ was used while for PHBV, a distance of $10 \mathrm{~cm}$ was used. The syringe needles were charged to $+17 \mathrm{kV}$ and polymer solutions spun onto an earthed rotating (300 RPM) aluminium foil coated mandrel. PLA and PHBV were setup in separate syringe pumps on either side of the rotating mandrel and charged by individual power supplies. Tri-layers were spun by sequentially spinning first $8 \mathrm{ml}$ PLA then $4 \mathrm{ml}$ of PHBV was simultaneously spun with $4 \mathrm{ml}$ PLA from a matching spinning setup on the other side of the mandrel then $8 \mathrm{ml}$ PLA was spun alone, creating a PLA-PHBVPLA tri-layer construct.

\section{Preparation of DED from EuroSkin}

We have previously shown that tissue engineered skin can be prepared from either cadaveric skin treated in-house or from the Dutch Tissue Bank, Euro Skin purchased commercially (22). Accordingly in this study we used Euroskin as our source of cadaveric dermis. Euroskin was purchased from Euro Skin Bank- Netherlands after seeking approval to use this material for research purposes.

The glycerol preserved Euroskin was washed several times with PBS until the glycerol was washed away. This was then de-epidermised by immersion in 1-M sodium chloride for 24 hours, and the epidermis was removed with gentle scraping with forceps. Euroskin was then washed thoroughly with PBS and then placed in 
medium at $37{ }^{0} \mathrm{C}$ for 2-3 days to confirm sterility. It was then incubated rehydrated in antibiotic-free media for $48 \mathrm{~h}$ prior to use.

\section{Isolation and culture of oral keratinocytes and fibroblasts}

Oral biopsies comprising of $0.5 \mathrm{~cm}^{2}$ of tissue were taken after informed consent and ethical approval from healthy volunteers or patients undergoing buccal mucosa removal for urethroplasty or for dental procedures (003463/2015). Specimen washed with PBS containing $100 \mathrm{mg} / \mathrm{mL}$ streptomycin and $100 \mathrm{IU} / \mathrm{mL}$ penicillin. The tissue samples were then incubated overnight $\left(12-18 \mathrm{~h}\right.$ ) at $4{ }^{0} \mathrm{C}$ in $0.1 \% \mathrm{w} / \mathrm{v}$ trypsin solution. Epidermal and dermal layers were peeled apart, and keratinocytes were isolated from the lower surface of the epidermis and the upper surface of the dermis by gentle scraping with a scalpel blade. Keratinocytes were then seeded at a density of $2 \times 10^{6}$ per T75 culture flask pre-seeded for $24 \mathrm{~h}$ with $5 \times 10^{5}$ i3T3 cells in Greens' keratinocyte medium; (DMEM) and Ham's F12 medium in a 3:1 ratio supplemented with $10 \%$ FCS, $10 \mathrm{ng} / \mathrm{mL}$ EGF, $0.4 \mu \mathrm{g} / \mathrm{mL}$ hydrocortisone, $10^{-10} \mathrm{~mol} / \mathrm{mL}$ cholera toxin, $1.8 \times 10^{-4} \mathrm{~mol} / \mathrm{L}$ adenine, $5 \mu \mathrm{g} / \mathrm{mL}$ insulin, $2 \times 10^{-3} \mathrm{~mol} / \mathrm{L}$ glutamine, $2 \times 10^{-7}$ $\mathrm{mol} / \mathrm{L}$ triiodothyronine, $0.625 \mu \mathrm{g} / \mathrm{mL}$ amphotericin $\mathrm{B}, 100 \mathrm{IU} / \mathrm{mL}$ penicillin and 100 $\mu \mathrm{g} / \mathrm{mL}$ streptomycin (25). Keratinocytes were maintained in culture to passage 3 then used in experiments. Fibroblasts were isolated from the dermis remaining after keratinocyte isolation. The dermis was washed with PBS then finely minced and placed in $10 \mathrm{~mL}$ of $0.5 \% \mathrm{w} / \mathrm{v}$ collagenase $\mathrm{A}$ at $37{ }^{\circ} \mathrm{C}$ in a humidified $\mathrm{CO}_{2}$ incubator for 18-20 h. After centrifugation of the resulting cell suspension at $400 \mathrm{~g}$ for $10 \mathrm{~min}$ cells were then cultured in DMEM media supplemented with $10 \%$ FCS $2 \times 10^{-3} \mathrm{~mol} / \mathrm{L}$ glutamine, $1.25 \mu \mathrm{g} / \mathrm{mL}$ amphotericin B, $100 \mathrm{IU} / \mathrm{mL}$ penicillin, and $100 \mu \mathrm{g} / \mathrm{mL}$ streptomycin. Fibroblasts were used in experiments between passages 4 and 9 .

\section{Cell seeding on scaffolds}

Tri-layer (PLA-PHBV-PLA) and monolayer (PLA, PHBV) electrospun scaffolds (2 $\mathrm{cm} \times 2 \mathrm{~cm}$ ) were sterilized (using 70\% v/v ethanol in $\mathrm{dH} 2 \mathrm{O}$ ) for 10 minutes, washed with PBS, and placed in 6-well plates. A $1 \mathrm{~cm}$ diameter culture well was formed on the scaffold using a $1 \mathrm{~cm}$ diameter stainless steel ring and $1.5 \times 10^{5}$ oral fibroblasts seeded inside. Constructs were cultured for 2 days at $37{ }^{\circ} \mathrm{C}$ and $5 \% \mathrm{CO}_{2}$. Scaffolds were then turned over and $3 \times 10^{5}$ oral keratinocytes were seeded onto each scaffold in Green's medium. After a further 2 days of culture, scaffolds were cut to $1 \mathrm{~cm}^{2}$ and raised to an air-liquid interface (this was counted as day 1) on stainless steel grids and some were treated with $200 \mu \mathrm{g} / \mathrm{mL}$ beta-aminopropionitrile (BAPN) throughout the culture period of 28 days. Samples were run in triplicate and experiments were repeated three times for contraction and cell viability $(\mathrm{N}=9)$.

\section{Cell seeding on dermis prepared from Euroskin}

Dermis prepared from Euroskin was cut into $2 \times 2 \mathrm{~cm}^{2}$ pieces and each placed in a sixwell plate. Oral fibroblasts were seeded on the inside of a $1 \mathrm{~cm}$ diameter stainless

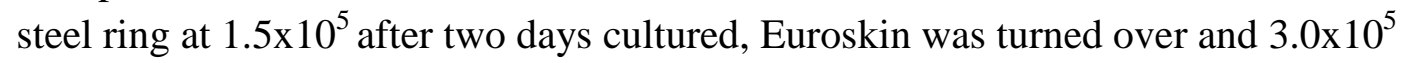
oral keratinocytes were seeded. Grafts were cultured for $48 \mathrm{~h}$ submerged, and then cut 
to $1 \mathrm{~cm}^{2}$ diameter and raised to air-liquid interface for the remainder of the assessment period. Samples were run in triplicate and experiments were repeated three times for contraction and cell viability $(\mathrm{N}=9)$.

Measurement of contraction of scaffolds

Contraction in vitro was assessed by serial digital photography cell seeded scaffolds and cell free scaffolds for 3 times a week until end of experiment. Images were analysed using ImageJ software (National Institutes of Health, Bethesda, MD, USA).

\section{Assessment of cell viability}

Cell metabolic activity was measured by a resazurin assay. Before air-liquid interface samples were washed with phosphate- buffered saline (PBS) and $5 \mathrm{~mL}$ of $50 \mu \mathrm{g} / \mathrm{mL}$ resazurin in PBS was added. Samples were incubated for $60 \mathrm{~min}$ at $37^{\circ} \mathrm{C}$. Then absorbance at $570 \mathrm{~nm}$ was measured in a colourimetric plate reader (Bio-TEK, NorthStar Scientific Ltd, Leeds, UK). Samples were then washed with PBS and returned to culture conditions. Cell viability testing was repeated after 3, 7, 14, 21 and 28 days of culture.

Mechanical testing of scaffolds

A total of 6 samples each (N=6), tri-layer (PLA-PHBV-PLA), PLA, PHBV, and Euroskin were cut and the width and thickness were measured using a ruler and micrometer respectively. These scaffolds were then clamped on a tensiometer (BOSE Electroforce Test Instruments). A load of $22 \mathrm{~N}$ and a ramp test at a rate of $0.1 \mathrm{~mm} / \mathrm{s}$ was applied. The strain was normalized to the length of the samples. The stress was normalized by the area (width $\mathrm{x}$ thickness). The first failure point or plateau was used to calculate the ultimate tensile strength (UTS) and the displacement at this point for the ultimate tensile strain (UT Strain). The Young's Modulus (YM) was then calculated.

Scanning electron cicroscopy (SEM) of scaffolds

The morphology of prepared PLA, PHBV and Trilayer (PLA-PHBV-PLA) scaffolds were observed through SEM (gold sputtering- Emscope SC 500A Sputter Coater) which was imaged with a FEI Inspect F scanning electron microscope (Cambridge, UK).

Fibre diameters and pore sizes were quantified from SEM images of electrospun scaffolds. From each sample (N=6), 4 images were selected and 10 fibres and 5 pores per image analysed for fibre diameter and pore size, respectively. This gave a total of 240 fibres and 120 pores analysed per scaffold. The software ImageJ (National Institutes of Health) was used for quantification. Pores were identified as areas of void space bounded by fibres on all sides at or near the same depth of field.

Histological evaluation of scaffolds

Scaffolds were embedded with OCT compound and frozen in liquid nitrogen. Scaffold cross-sections (5- or 10- $\mu \mathrm{m}$ thickness) were cut with a cryostat (Leica CM300) and placed on frosted slides. The sample-carrying slides were then soaked in 
deionised water (2 min) to remove the OCT, stained with haematoxylin (1.5 min), and afterwards washed with running tap water (4 min) and stained with eosin (5 min). After another wash with tap water (5 min), samples were dehydrated in $70 \%$ alcohol, followed with the immersion in $100 \%$ alcohol. Finally, the slides were cleaned twice in xylene, and mounted with a coverslip by using a DPX mounting medium. After mounting slides were observed by a light microscope.

\section{Statistics}

The results were presented as the mean \pm standard deviation (SD). Data were analysed with Independent-Sample T Test and analysis of variance (ANOVA) to determine the difference among groups using SPSS 23 software programme. Differences in mechanical properties were statistically tested with a GraphPad Prism 8 software using a Friedman test and doing multiple comparisons between individual groups using a Dunn's test. A p value $<0.05$ was considered statistically significant.

\section{Results}

Characterization of scaffolds and cells cultured on scaffolds

SEM and histological images of the scaffolds are shown in Figure 1A and 1B. The mean fibre diameters and pore sizes of PLA and PHBV scaffolds were $2.4 \pm 0.77$ and $0.85 \pm 0.21 \mu \mathrm{m}$ for fibre diameter respectively $(\mathrm{p}<0.001)$ and $10.8 \pm 2.3$ and $4.3 \pm 1.1 \mu \mathrm{m}$ for pore size respectively $(\mathrm{p}<0.001)$. Porosity measurements showed a significant difference between the microfibrous scaffolds (as shown in 1A (A) and the nanofibrous scaffolds (shown in 1A(B) with the microfibrous PLA being around 28\% more porous. Figure 1A (E) shows a cross section through this trilayer scaffold in which it can be seen that the overall thickness of the scaffold was around $400 \mu \mathrm{m}$ while the thickness of the PHBV layer was around $30 \mu \mathrm{m}$.

Figure 1A also shows culture of oral fibroblasts on the lower surface of the trilayer scaffold (D) and of oral keratinocytes on the upper surface of the trilayer scaffold (F). Figure 1B shows H\&E histology of oral keratinocytes and fibroblasts cultured on tissue engineered buccal mucosa made based on Euroskin (A) versus tissue engineered buccal mucosa made using the trilayer of PLA/PHBV/PLA. This shows a decent number of fibroblasts in the lower layer and a much thicker layer of keratinocytes on the upper surface for both.

Mechanical properties of the scaffolds

Figure 2 compares the YM, UTS and UT Strain of Euroskin and three synthetic scaffolds. There are significant differences between the groups shown by one-way ANOVA $(\mathrm{p}<0.001)$ and the tables in Figure 2 show multiple statistical comparisons between these.

Compared to Euroskin, UT Strain was not significantly different for any of the three synthetic scaffolds. However, the UTS and YM of PLA samples were significantly lower than that of Euroskin ( $<<0.001$ and $\mathrm{p}<0.05$ respectively). While these two properties were not significantly different for PHBV samples compared to Euroskin, only the UTS of the trilayer samples was significantly lower $(\mathrm{p}<0.05)$. 


\section{Cell viability on scaffolds}

Figure 3 shows cell viability of the cells on the scaffolds assessed throughout 28 days of culture using a resazurin salt assay. Metabolic activity was significantly increased for all groups over time without there being any significant differences between cells grown on the different scaffolds. Additionally there was no effect of the addition of $200 \mu \mathrm{m} \beta$-APN on cell viability for cells growing on these scaffolds. The figure also shows that there was no evidence any metabolic activity for Euroskin cultured on its own.

\section{Contraction of scaffolds by keratinocytes and fibroblasts}

Figure 4 shows the appearance of these buccal mucosa constructs at day 1 and day 28 when based on Euroskin showing extensive contraction which is slightly reduced by the addition of $\beta$-APN. In contrast cells on the trilayer show little contraction by day 28 and the addition of $\beta$-APN was without effect.

Figure 5 shows the extent to which the cells on different scaffolds contracted the scaffold and the effect of $200 \mu \mathrm{m} \beta$-APN on this. It was very evident that as previously reported the keratinocytes and fibroblasts contracted the tissue engineered constructs when these were based on natural dermis from Euroskin. The addition of $\beta$ APN significantly reduced contraction of these scaffolds $(\mathrm{p}<0.01)$.

For the other three scaffolds, PLA, PHBV and the trilayer, the extent of contraction over 28 days was much less and was not significantly affected by the addition of $200 \mu \mathrm{g} / \mathrm{ml} \beta$-APN. These results are also summarised in Table 1.

\section{Discussion}

The need for tissue engineered grafts was identified for those patients who require treatment for recurrent long complex urethral strictures (26). In the initial treatment of long complex urethral strictures autologous buccal mucosa was shown to be successful. However, the urethral re-stricture rate remains significant at 14.5-15.7\% (27). Treatment of recurrent strictures can be more difficult because of urethral shortening from prior surgery, the degree of inflammation of the urethra and the spondiofibrotic fibrosis and periurethral scarring itself and associated poor blood supply. For these challenging patients it may be possible to harvest further autologous buccal tissue but with increasing likelihood of donor site complications. In some cases because of prior surgery this may not be possible. Accordingly in recent years, tissue engineered buccal mucosa has been explored for urethral reconstruction. Our previous studies looking at tissue engineered skin and exploring the contraction seen in this have identified that it is the epithelial cells rather than the stromal fibroblasts which drive the contraction by stimulating the enzyme lysyl oxidase which crosslinks collagen fibres (28). A pharmacological study revealed that an inhibitor of lysyl oxidase, $\beta$-APN (found in the sweet-pea family) was capable of significantly reducing contraction. We have also previously explored a few other routes to block or resist contraction. The most promising of these was to suture the graft in place against a rigid framework in the laboratory for the first 7 days and this seems to reduce the extent of the contraction (29). 
The aim of this study was to explore alternative synthetic scaffolds as substrates for the production of tissue engineered buccal mucosa for future use in reconstruction of the urethra. As part of this we explored the addition of an inhibitor of the collagen crosslinking enzyme lysyl oxidase $\beta$-APN to see to what extent this might reduce the cell induced contraction of these grafts in vitro.

The main findings of this study were that it proved possible to make a trilayer scaffold out of microfibers of PLA and nanofibers of PHBV which supported the production of a tissue engineered buccal mucosa. The mechanical properties of the trilayer were comparable to those of tissue engineered buccal mucosa based on native dermis but encouragingly, cells showed relatively little ability to contraction these in vitro. We found $\beta$-APN reduced the contraction of the construct when cells were cultured on native dermal collagen but had no effect on the very slight degree of contraction seen when the cells were cultured on the trilayer scaffold.

In this study, we designed a scaffold based on a combination of polylactic acid and PHBV. PLA is FDA approved synthetic biodegradable and biocompatible polymer of lactic acid produced by ring opening polymerisation of lactic acid. It is a hydrophobic, semi-crystalline and amorphous polymer (30). It has been used as a biomaterial for a variety of different urological applications $(18,19,31,32)$. In our comparisons we studied electrospun PLA on its own and showed that it is a good substrate for the growth of oral keratinocytes and fibroblasts. However, it has relatively poor mechanical properties compared to Euroskin. Cells grown on PLA scaffolds showed approximately $25 \%$ contraction.

The second polymer, PHBV, is a natural biodegradable polymer which is formed by bacterial fermentation and it has been electrospun into fibrous scaffolds for a wide range of applications $(33,34)$ including skin grafts, drug-delivery systems, cartilage tissue engineering, and reconstructive implants (35-38). It is relatively hydrophobic with a longer and much slower rate of degradation which can be seen as disadvantages (39). It is often used in combination with other polymers to obtain new materials (40).

We developed a trilayer scaffold in which PHBV was used to produce a pseudo-basement membrane of nanofibers, separating oral keratinocytes from oral fibroblasts. We previously demonstrated (23) that a nanofibrous scaffold can act as a barrier for cell penetration while a microfibrous scaffold provides cell attachment and proliferation for two different cell types.

With respect to the ultimate tensile strength the trilayer was $0.13 \pm 0.035$ compared to $2.34 \pm 0.67$ for Euroskin. We suggest it is not essential that the mechanical properties are exactly as for the native tissue but that they are "good enough" to maintain a patent urethra long-term.

However the trilayer scaffolds presented the closest values for stiffness and stretchability compared to that of Euroskin. The addition of the nanofibrous component of the trilayer made a material with mechanical properties closer to native tissues and it was easy to handle. By this we mean that it could be picked up, shaped and handled without tangling, sticking or loss of shape. 
In a previous study, we tried to use oral mucosal cells seeded on a synthetic biodegradable scaffold made of polylactic-co-glycolic acid (PLGA) for urethral reconstruction (14). However, all scaffolds showed extensive contraction (more than $50 \%$ over 14 days) irrespective of the method of sterilization or the presence of cells. This was clearly unsatisfactory.

Another group, Wei et al., prepared a polycaprolactone/ silk/collagen nano-3D porous scaffold for urethral reconstruction. They showed that oral mucosal epithelial cells grew well on the scaffold. However these authors did not comment on scaffold contraction and evaluated cell viability for only seven days (41). Another study evaluated the capacity of a PLA scaffold seeded with rabbit adipose tissue-derived stem cells to repair a urethral defect in a rabbit model. After 4 or 6 weeks of implantation, the grafts were collected and analysed histologically. Their data demonstrated good integration into native tissues and they concluded that cell-seeded PLA based materials would be a good candidate for the next generation of TE urethras (42).

Atlantida Raya- Rivera et al., reported the outcomes of five male patients who underwent urethroplasty. They took bladder tissue biopsy from each patient, and cells were expanded and seeded onto tubularised PLGA scaffolds. 4-6 cm manufactured scaffolds were used for urethral reconstruction. After 71 months, median follow serial radiographic, urinary flow rate, and endoscopic studies showed the maintenance of wide urethral calibres without strictures. The authors concluded that tubularised urethra can be engineered to be used as a prospective clinical procedure. However, this was just a small clinical trial that required an invasive bladder biopsy using a suprapubic incision which carries risks of serious complications (43). Our group has experience on contraction of TE skin and we have previously demonstrated that extensive collagen crosslinking is related to contraction (28). This can be reduced by the inhibition of lysyl oxidase, a key enzyme involved in collagen crosslinking (29). The current study showed that $\beta$-APN significantly inhibited Euroskin contraction, however this effect was not seen in PLA scaffolds. This may be explained by the lower collagen levels found in the PLA scaffolds compared to the natural dermal substrates.

In this study, we successfully seeded oral keratinocytes and fibroblasts on each side respectively of PLA-PHBV-PLA micro-nano-3D porous scaffolds. Cells grew well on both PLLA sides of the membrane and the PHBV barrier membrane provided a barrier against cell infiltration between the different cells on the PLLA scaffolds either side of the barrier.

A limitation of this study is that while it is ideal for looking at short-term contraction over several weeks in vitro it cannot shed light on the complex issue of fibrosis which can occur many months post implantation. In our study it occurred in two patients eight and nine months post implantation strongly (12) suggesting that this is an immune system triggered process. These patients suffered from recurrent fibrosis throughout the previous 10 years before transplantation of tissue engineered buccal 
mucosa. The other patients maintained luminal patency throughout the following nine years of follow-up (13).

It might in the future be possible to deliver a lysyl oxidase inhibitor as part of a drug eluting scaffold but we have to point out that there are very few studies as yet looking at the process of fibrosis post implantation of tissue engineered materials. The fact that this can occur many months post implantation suggests that this will be a challenge to study and it may not be a process which can be studied in animal models if the triggering is an underlying recurrent inflammatory process.

\section{Conclusion}

A PLA-PHBV-PLA trilayer micro-nano-3D porous scaffold is suitable for oral keratinocyte and fibroblast growth with good cell viability and minimal contraction. This cell loaded material also has good mechanical properties and histological analyses showed its ability to mimic normal human oral mucosal morphology for TE urethral reconstruction. Furthermore trilayer scaffolds have the advantage over biological scaffolds for avoiding immunological rejection and preventing viral infections. Further investigations are necessary to translate this in vitro technology into the preclinical and clinical studies. 


\section{References}

1. Lumen N, Hoebeke P, Willemsen P, De Troyer B, Pieters R, Oosterlinck W. Etiology of urethral stricture disease in the 21st century. The Journal of urology 2009; 182: 983-7.

2. Fenton AS, Morey AF, Aviles R, Garcia CR. Anterior urethral strictures: etiology and characteristics. Urology 2005; 65: 1055-8.

3. Anger JT, Buckley JC, Santucci RA, Elliott SP, Saigal CS. Trends in stricture management among male Medicare beneficiaries: underuse of urethroplasty? Urology 2011; 77: 481-5.

4. Santucci RA, Joyce GF, Wise M. Male urethral stricture disease. The Journal of urology 2007; 177: 1667-74.

5. Lumen N, Oosterlinck W, Hoebeke P. Urethral reconstruction using buccal mucosa or penile skin grafts: systematic review and meta-analysis. Urologia internationalis 2012; 89: 387-94.

6. Barbagli G, Sansalone S, Djinovic R, Romano G, Lazzeri M. Current controversies in reconstructive surgery of the anterior urethra: a clinical overview. International braz j urol : official journal of the Brazilian Society of Urology 2012; 38: 307-16; discussion 16.

7. Barbagli G. When and how to use buccal mucosa grafts in penile and bulbar urethroplasty. Minerva urologica e nefrologica $=$ The Italian journal of urology and nephrology 2004; 56: 189-203.

8. Markiewicz MR, Lukose MA, Margarone JE, Barbagli G, Miller KS, Chuang SK. The oral mucosa graft: a systematic review. The Journal of urology 2007; 178: 387-94.

9. Jang TL, Erickson B, Medendorp A, Gonzalez CM. Comparison of donor site intraoral morbidity after mucosal graft harvesting for urethral reconstruction. Urology 2005; 66: 716-20.

10. Das SK, Kumar A, Sharma GK et al. Lingual mucosal graft urethroplasty for anterior urethral strictures. Urology 2009; 73: 105-8.

11. Bhargava S, Chapple CR, Bullock AJ, Layton C, MacNeil S. Tissueengineered buccal mucosa for substitution urethroplasty. BJU Int 2004; 93: 807-11. 12. Bhargava S, Patterson JM, Inman RD, MacNeil S, Chapple CR. Tissueengineered buccal mucosa urethroplasty-clinical outcomes. Eur Urol 2008; 53: 12639.

13. Osman NI, Patterson JM, MacNeil S, Chapple CR. Long-term follow-up after tissue-engineered buccal mucosa urethroplasty. Eur Urol 2014; 66: 790-1.

14. Selim M, Bullock AJ, Blackwood KA, Chapple CR, MacNeil S. Developing biodegradable scaffolds for tissue engineering of the urethra. BJU Int 2011; 107: 296302.

15. Harrison CA, Heaton MJ, Layton CM, Mac Neil S. Use of an in vitro model of tissue-engineered human skin to study keratinocyte attachment and migration in the process of reepithelialization. Wound repair and regeneration : official publication of 
the Wound Healing Society [and] the European Tissue Repair Society 2006; 14: 2039.

16. Engelhardt EM, Micol LA, Houis S et al. A collagen-poly(lactic acid-covarepsilon-caprolactone) hybrid scaffold for bladder tissue regeneration. Biomaterials 2011; 32: 3969-76.

17. Telemeco TA, Ayres C, Bowlin GL et al. Regulation of cellular infiltration into tissue engineering scaffolds composed of submicron diameter fibrils produced by electrospinning. Acta biomaterialia 2005; 1: 377-85.

18. Roman S, Mangir N, Bissoli J, Chapple CR, MacNeil S. Biodegradable scaffolds designed to mimic fascia-like properties for the treatment of pelvic organ prolapse and stress urinary incontinence. Journal of biomaterials applications 2016; 30: 1578-88.

19. Mangir N, Bullock AJ, Roman S, Osman N, Chapple C, MacNeil S. Production of ascorbic acid releasing biomaterials for pelvic floor repair. Acta biomaterialia 2016; 29: 188-97.

20. Sharma K, Bullock A, Ralston D, MacNeil S. Development of a one-step approach for the reconstruction of full thickness skin defects using minced split thickness skin grafts and biodegradable synthetic scaffolds as a dermal substitute. Burns : journal of the International Society for Burn Injuries 2014; 40: 957-65. 21. Mangera A, Bullock AJ, Roman S, Chapple CR, MacNeil S. Comparison of candidate scaffolds for tissue engineering for stress urinary incontinence and pelvic organ prolapse repair. BJU Int 2013; 112: 674-85.

22. Deshpande P, Ramachandran C, Sefat F et al. Simplifying corneal surface regeneration using a biodegradable synthetic membrane and limbal tissue explants. Biomaterials 2013; 34: 5088-106.

23. Frazer J. Byea JB, Leanne Blacka, Anthony J. Bullocka, Sasima Puwanuna, Keyvan Moharamzadehb, Gwendolen C. Reillya, Anthony J. Ryanc and Sheila MacNeil. Development of bilayer and trilayer nanofibrous/microfibrous scaffolds for regenerative medicine. Biomaterials Science 2013; 1: 942-51.

24. Bye Frazer J BAJ, Singh Rita, Sefat Farshid, Roman Sabiniano, MacNeil Sheila. Development of a Basement Membrane Substitute Incorporated Into an Electrospun Scaffold for 3D Skin Tissue Engineering. Journal of Biomaterials and Tissue Engineering 2014; 4: 686-92.

25. Rheinwald JG, Green H. Serial cultivation of strains of human epidermal keratinocytes: the formation of keratinizing colonies from single cells. Cell 1975; 6: 331-43.

26. Chapple C, Andrich D, Atala A, et al. SIU/ICUD Consultation on Urethral Strictures: The management of anterior urethral stricture disease using substitution urethroplasty. Urology 2014; 83: S31-47.

27. Wessells H, McAninch JW. Current controversies in anterior urethral stricture repair: free-graft versus pedicled skin-flap reconstruction. World journal of urology 1998; 16: 175-80. 
28. Harrison CA, Gossiel F, Layton CM, et al. Use of an in vitro model of tissueengineered skin to investigate the mechanism of skin graft contraction. Tissue engineering 2006; 12: 3119-33.

29. Patterson JM, Bullock AJ, MacNeil S, Chapple CR. Methods to reduce the contraction of tissue-engineered buccal mucosa for use in substitution urethroplasty. Eur Urol 2011; 60: 856-61.

30. Kulkarni RK, Pani KC, Neuman C, Leonard F. Polylactic acid for surgical implants. Archives of surgery (Chicago, Ill. : 1960) 1966; 93: 839-43.

31. Fu WJ, Zhang X, Zhang BH, et al. Biodegradable urethral stents seeded with autologous urethral epithelial cells in the treatment of post-traumatic urethral stricture: a feasibility study in a rabbit model. BJU Int 2009; 104: 263-8.

32. Xu Y, Fu W, Wang Z, Li G, Zhang X. A tissue-specific scaffold for tissue engineering-based ureteral reconstruction. PloS one 2015; 10: e0120244.

33. Tong HW, Wang M, Li ZY, Lu WW. Electrospinning, characterization and in vitro biological evaluation of nanocomposite fibers containing carbonated hydroxyapatite nanoparticles. Biomedical materials (Bristol, England) 2010; 5: 054111.

34. Bahari Javan N, Rezaie Shirmard L, Jafary Omid N, Akbari Javar H, Rafiee Tehrani M, Abedin Dorkoosh F. Preparation, statistical optimisation and in vitro characterisation of poly (3-hydroxybutyrate-co-3-hydroxyvalerate)/poly (lactic-coglycolic acid) blend nanoparticles for prolonged delivery of teriparatide. Journal of microencapsulation 2016: 1-15.

35. Li H, Chang J. Fabrication and characterization of bioactive wollastonite/PHBV composite scaffolds. Biomaterials 2004; 25: 5473-80.

36. Kose GT, Korkusuz F, Ozkul A et al. Tissue engineered cartilage on collagen and PHBV matrices. Biomaterials 2005; 26: 5187-97.

37. Li H, Chang J. Preparation, characterization and in vitro release of gentamicin from PHBV/wollastonite composite microspheres. Journal of controlled release : official journal of the Controlled Release Society 2005; 107: 463-73.

38. Ito Y, Hasuda H, Kamitakahara M et al. A composite of hydroxyapatite with electrospun biodegradable nanofibers as a tissue engineering material. Journal of bioscience and bioengineering 2005; 100: 43-9.

39. Yoon YI, Moon HS, Lyoo WS, Lee TS, Park WH. Superhydrophobicity of PHBV fibrous surface with bead-on-string structure. Journal of colloid and interface science 2008; 320: 91-5.

40. Li X, Liu KL, Wang M et al. Improving hydrophilicity, mechanical properties and biocompatibility of poly[(R)-3-hydroxybutyrate-co-(R)-3-hydroxyvalerate] through blending with poly[(R)-3-hydroxybutyrate]-alt-poly(ethylene oxide). Acta biomaterialia 2009; 5: 2002-12.

41. Wei G, Li C, Fu Q, Xu Y, Li H. Preparation of PCL/silk fibroin/collagen electrospun fiber for urethral reconstruction. International urology and nephrology 2015; 47: 95-9. 
42. Wang DJ, Li MY, Huang WT et al. Repair of urethral defects with polylactid acid fibrous membrane seeded with adipose-derived stem cells in a rabbit model. Connective tissue research 2015; 56: 434-9.

43. Raya-Rivera A, Esquiliano DR, Yoo JJ, Lopez-Bayghen E, Soker S, Atala A. Tissue-engineered autologous urethras for patients who need reconstruction: an observational study. Lancet (London, England) 2011; 377: 1175-82. 


\section{Figures and Tables}

Fig. 1A. Scanning electron micrograph (SEM) of electrospun scaffolds. (A) PLA; (B) PHBV; (C) trilayer (PLA-PHBV-PLA); (D) oral fibroblast cells are tightly adhered on the lower surface of the trilayer and growing well; $(\boldsymbol{E})$ cross-section of trilayer; $(\boldsymbol{F})$ oral keratinocytes are grown and stretched well on the upper layer of the trilayer scaffolds.

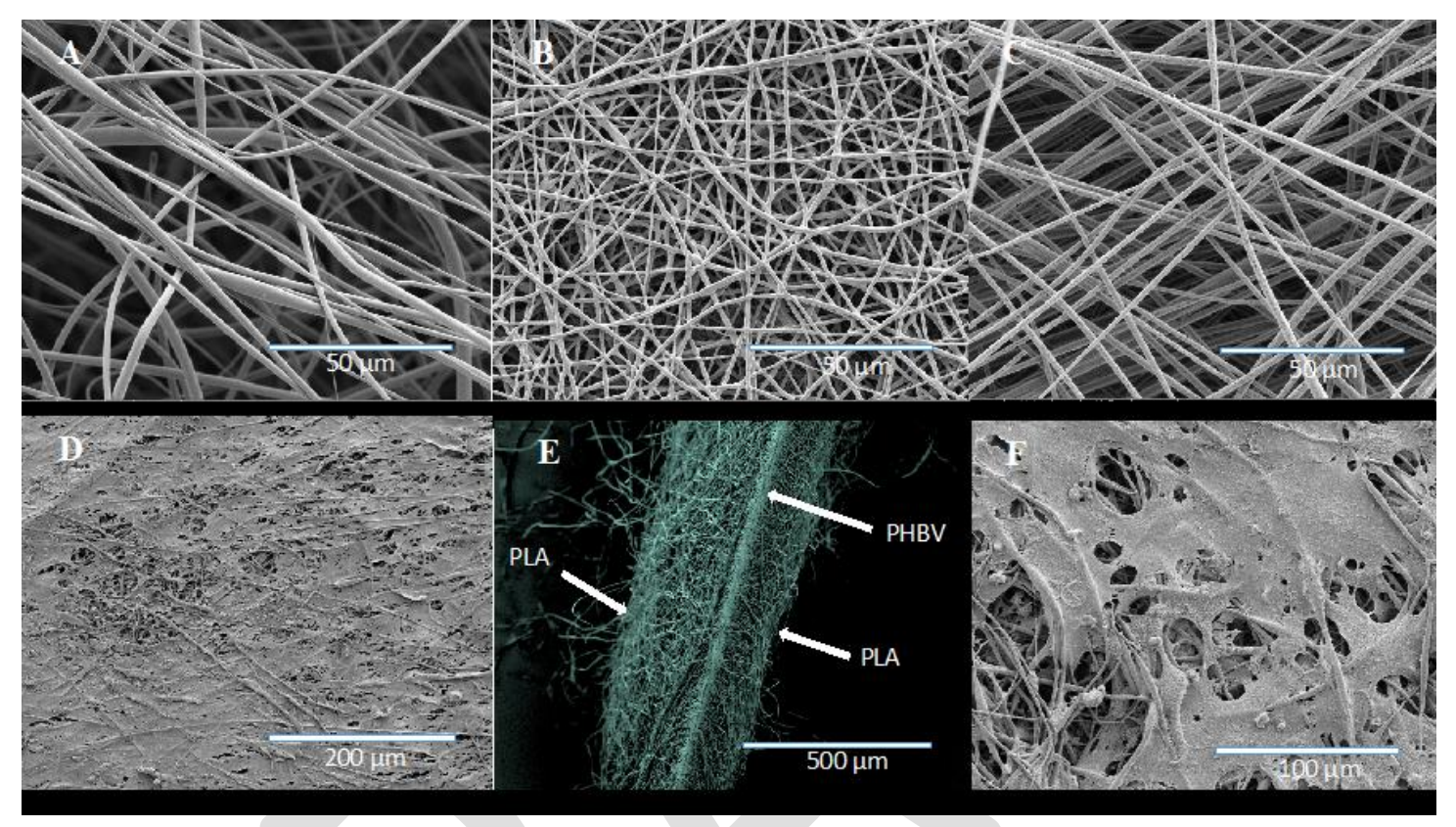

Fig. 1B. Histological appearance of tissue-engineered buccal mucosa using (left) Euroskin; and (right) trilayer (PLA-PHBV-PLA). Oral keratinocytes were seeded on the upper surface and fibroblasts were seeded on the lower surface of the scaffolds and stained with haematoxylin and eosin after culture periods (magnification x10)

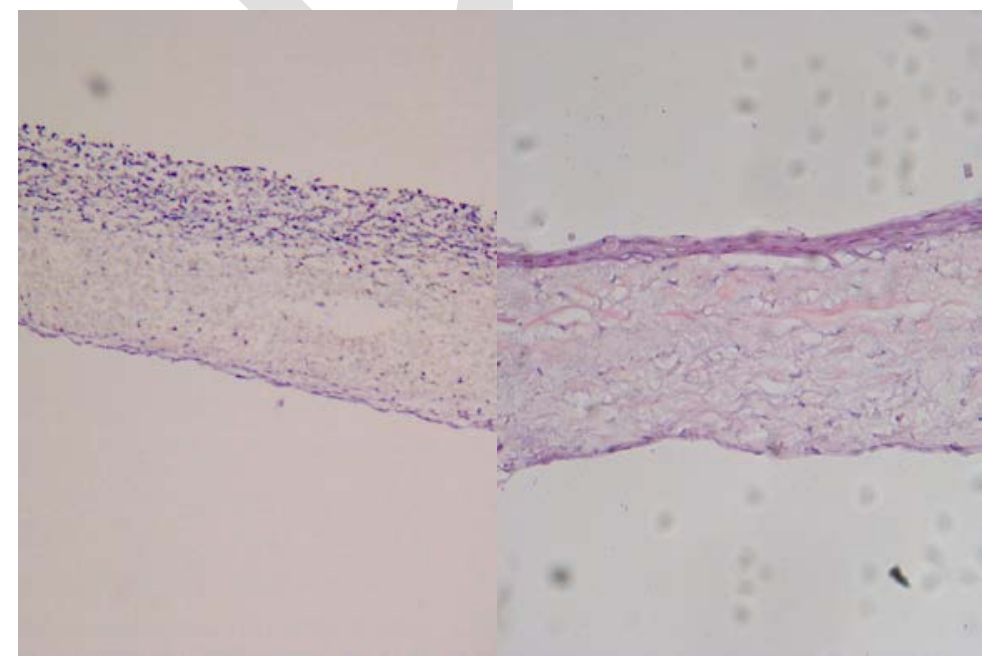


Fig. 2. Mechanical properties mean \pm standard deviation (SD) values for Young's modulus, tensile strength, and strain at ultimate tensile strength.
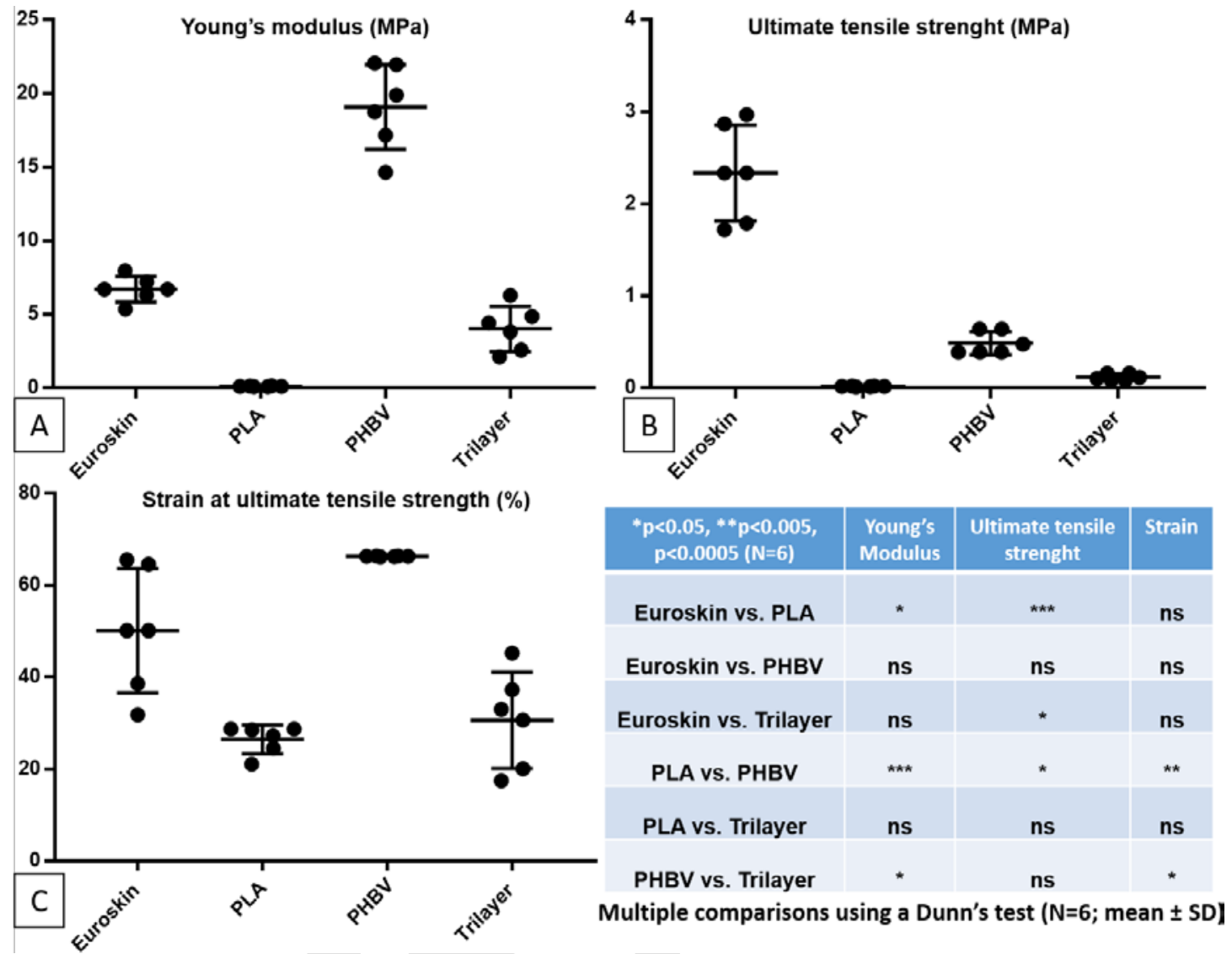

Fig. 3. Cell viability of oral keratinocytes and fibroblasts gown on scaffolds. BAPN: beta-aminopropionitrile.

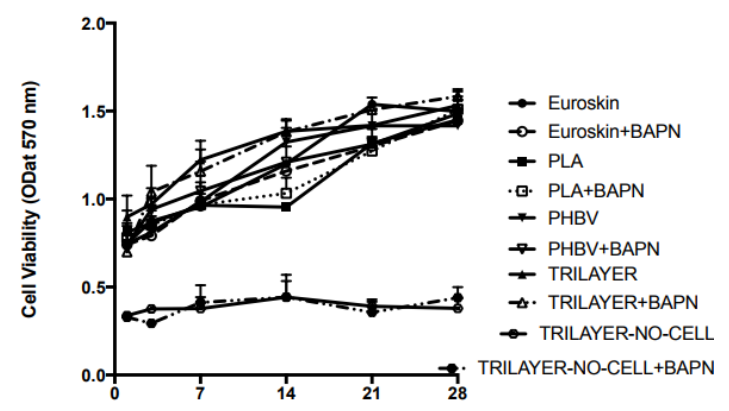


Fig. 4. Appearance of tissue engineered scaffolds at Day $1(\boldsymbol{A}, \boldsymbol{E})$ and Day $28(\boldsymbol{B}, \boldsymbol{F})$ is based on Euroskin and Trilayer, in the absence $(A, B, E, F)$ and presence $(C, D, G$, $\boldsymbol{H})$ of beta-amino propionitrile ( $\beta$-APN).

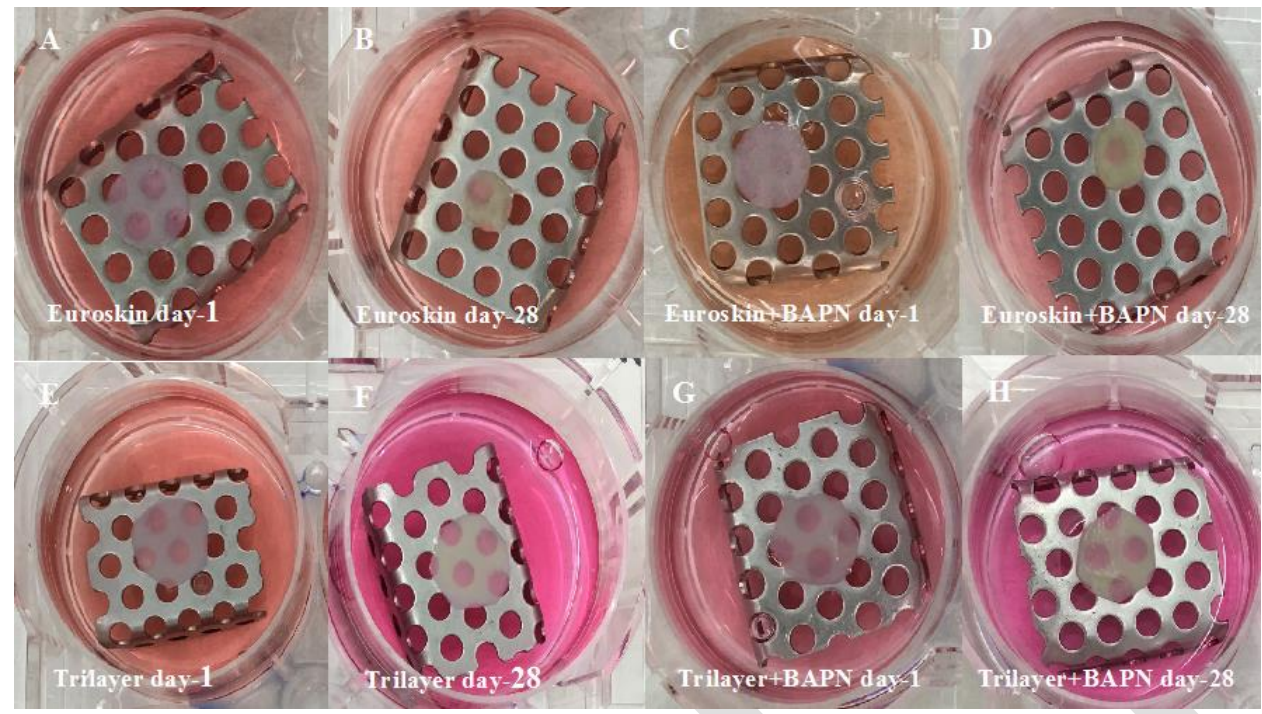

Fig. 5. Time course of scaffold contraction. BAPN: beta-aminopropionitrile.
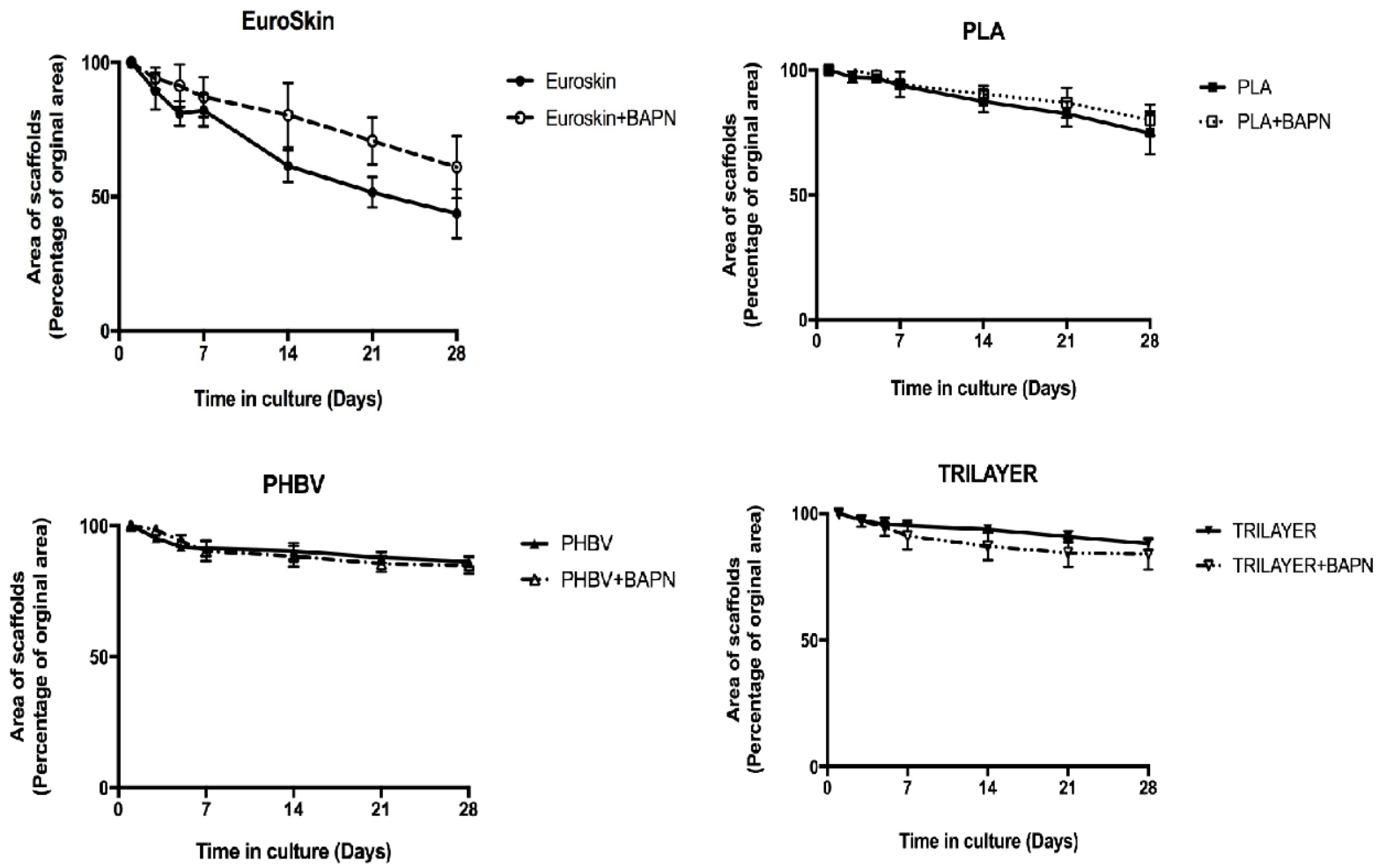


\begin{tabular}{|c|c|c|c|c|c|c|c|}
\hline Group & Day 1 & Day 3 & Day 5 & Day 7 & Day 14 & Day 21 & Day 28 \\
\hline Euroskin & 100 & $89.4 \pm 6.9$ & $81.1 \pm 4.6$ & $82.2 \pm 6.0$ & $61.4 \pm 6.1$ & $51.6 \pm 5.7$ & $43.6 \pm 9.1$ \\
\hline Euroskin + BAPN & 100 & $94.1 \pm 4.1$ & $91.3 \pm 8.0$ & $87.1 \pm 7.4$ & $80.3 \pm 11.9$ & $70.8 \pm 8.1$ & $61.1 \pm 11.6$ \\
\hline PLA & 100 & $97.2 \pm 2.3$ & $96.7 \pm 0.9$ & $93.7 \pm 1.2$ & $87.3 \pm 4.3$ & $82.4 \pm 5.0$ & $74.7 \pm 8.4$ \\
\hline PLA+BAPN & 100 & $99.8 \pm 3.1$ & $97.8 \pm 2.5$ & $94.2 \pm 5.1$ & $90.3 \pm 3.3$ & $86.9 \pm 6.1$ & $80.1 \pm 6.1$ \\
\hline PHBV & 100 & $95.2 \pm 1.5$ & $91.9 \pm 1.6$ & $91.3 \pm 2.9$ & $89.9 \pm 3.1$ & $87.8 \pm 2.1$ & $86.3 \pm 2.1$ \\
\hline PHBV+BAPN & 100 & $98.3 \pm 1.2$ & $94.1 \pm 2.3$ & $90.2 \pm 3.8$ & $88.2 \pm 3.9$ & $85.4 \pm 3.1$ & $84.8 \pm 88.1$ \\
\hline TRILAYER & 100 & $97.4 \pm 1.8$ & $95.6 \pm 0.5$ & $95.2 \pm 2.2$ & $93.7 \pm 1.0$ & $90.8 \pm 2.2$ & $88.1 \pm 2.4$ \\
\hline TRILAYER+BAPN & 100 & $97.1 \pm 2.3$ & $94.7 \pm 3.6$ & $91.1 \pm 5.6$ & $87.0 \pm 5.5$ & $86.8 \pm 6.0$ & $86.5 \pm 7.5$ \\
\hline
\end{tabular}

Values are mean \pm standard deviation (SD). The contraction of the scaffolds were calculated by dividing the area of each scaffold at the termination of the culture period by its original area (first day area). (Area*100/original area). 